\title{
Correction to: Acute Sets of Exponentially Optimal Size
}

\author{
Balázs Gerencsér $^{1,2}$ (D) Viktor Harangi ${ }^{1}$
}

\section{Correction to: Discrete Comput Geom https://doi.org/10.1007/s00454-018-9985-0}

Due to a typesetting error some mistakes were introduced; most importantly at the end the third page of the article in the displayed equation $\left(\frac{N}{2}\right)$ should read $\left(\begin{array}{c}N \\ 2\end{array}\right)$. Other minor formatting improvements have been made which should not alter the meaning. The original article has been updated to correct the errors.

The original article can be found online at https://doi.org/10.1007/s00454-018-9985-0.

Balázs Gerencsér

gerencser.balazs@ renyi.mta.hu

Viktor Harangi harangi@ renyi.hu

1 MTA Alfréd Rényi Institute of Mathematics, Reáltanoda utca 13-15, Budapest 1053, Hungary

2 Department of Probability and Statistics, Eötvös Loránd University, Pázmány Péter sétány 1/c, 1117 Budapest, Hungary 